\title{
Innovative textile technology with antimicrobial activity: preliminary tests
}

\author{
Gaia Ortalli, ${ }^{1}$ Marco Soligo, ${ }^{2}$ Pietro Messori, ${ }^{2}$ Ester Oliva, ${ }^{1}$ Claudio Farina ${ }^{1}$ \\ ${ }^{1}$ UOC Microbiologia e Virologia,, Ospedale di Bergamo, ASST Papa Giovanni XXIII, Bergamo; ${ }^{2}$ Urogynecology Unit, \\ Department of Woman, Mother and Neonate, ASST Fatebenefratelli - Sacco, University of Milano, Italy
}

\begin{abstract}
Summary
The prevalence of urinary tract infections (UTI) and antibiotic resistance prompts researchers to develop strategies for bacterial containment, such as antiseptic-active textiles. We verified the in vitro antimicrobial effect of innovative Nexus Energy Fiber compared to conventional cotton fabrics. The analytical procedure is performed in accordance with ASTM E-2180-07 document. Tests are realized both against clinical and reference UTI-responsible microorganisms by comparing CFUs declines on the two textiles, at different contact times $(0,6,24,48$ hours $)$ and at different distances between the fabrics and the microbial strains $(0,0.5-1 \mathrm{~cm})$. Results show that Nexus Energy Fiber has a measurable bactericidal effect which depends on the distances and is directly proportional to the contact time on most of the tested microbial strains.
\end{abstract}

\section{Introduction}

The prevalence of urinary tract infections (UTI) and the problem of antibiotic resistance prompt researchers to develop innovative strategies for bacterial containment. The usage of textiles with intrinsic antiseptic properties can be an interesting solution to obtain containment of colonizing microorganisms (2). The innovative fabric Nexus Energy Fiber ${ }^{\circledR}$ (NEF), Italy shows excellent

Correspondence: Claudio Farina, UOC Microbiologia e Virologia, ASST “Papa Giovanni XXIII”, Piazza OMS 1, 24127 Bergamo, Italy. Tel.: +39.035.2673666 - Fax: +39.035.2674921.

E-mail: cfarina@asst-pg23.it

Key words: Urinary Tract Infection; textile; antimicrobial effect.

Contributions: the authors contributed equally.

Conflict of interest: the authors declare no potential conflict of interest.

Received for publication: 17 December 2017.

Accepted for publication: 19 December 2017.

CCopyright G. Ortalli et al., 2017

Licensee PAGEPress, Italy

Microbiologia Medica 2017; 32:7239

doi:10.4081/mm.2017.7239

This article is distributed under the terms of the Creative Commons Attribution Noncommercial License (by-nc 4.0) which permits any noncommercial use, distribution, and reproduction in any medium, provided the original author(s) and source are credited. static-eliminating properties due to its far infrared radiation physical activity added to the presence of silver compounds (3). Purpose of this study is to verify whether NEF has an in vitro antimicrobial effect compared to conventional pure cotton fabrics.

\section{Type of the study}

On top, its antimicrobial activity is evaluated on microorganisms responsible for UTI: particularly, E. coli, P. aeruginosa, E. faecalis and $S$. saprophyticus isolated from urine cultures of hospitalized patients. The reference microorganisms belong instead to the ATTC collection: S.aureus ATCC 6538 and K. pneumoniae ATCC 4352. All the analysis are performed in triplicate in accordance with the document ASTM E-2180-07 Determining the activity of antimicrobial agents incorporated into polymeric or hydrophobic materials (1).

Specifically, the test took place in two rounds. Round 1 (direct contact conditions) involved the evaluation of the antimicrobial response when the fabric is at direct contact with microbial cultures in exponential growth phase. A slurry agar was used (4). If any antimicrobial effect is measured, round 2 (remote conditions) was performed: the fabrics were placed $0.5-1 \mathrm{~cm}$ away from the microbial culture. For both tests, positive and negative control references were set up to demonstrate media reliability and to exclude possible contaminations. The antimicrobial response was tested at different contact times: 0, 6, 24 and 48 hours.

The bactericidal effect was estimated by comparing the different decline in the CFUs on pure cotton and on NEF. Antimicrobial effect percentages of NEFversus conventional pure cotton were calculated as indicated in paragraph 9 of the ASTM E-2180-07 (1).

Results are reported in Tables 1 and 2.

Direct contact tests proved that NEF has no antimicrobial effect against wild strains of E. faecalis and S. saprophyticus at any contact time. It presented a bactericidal response contra $P$. aeruginosa strain after 48 hours, while no effect was observed for shorter contact times. Significantly, it showed an almost total killing of $S$. aureus after a 6-hours long contact period. However, it disappeared if the contact time was increased up to 48 hours. A progressive increment of the antimicrobial effect against $K$. pneumoniae as a function of contact time was also observed. The results about $E$. coli clinical strain were similar: nonetheless, a peak of bactericidal power after a 24-hour contact time was detected.

\section{Conclusions}

In conclusion, NEF has a bactericidal power correlated to the contact time contra K. pneumoniae, E. coli and P. aeruginosa, it has 
Table 1. Antimicrobial effect percentages of Nexus Energy Fiber ${ }^{\circledR}$ versus ordinary pure cotton as a function of time, at direct contact conditions.

\begin{tabular}{lcccc} 
Microorganisms & \multicolumn{3}{c}{$\begin{array}{c}\text { Antimicrobial effect (\%) in function of time } \\
\text { 24 hours }\end{array}$} & 48 hours \\
Klebsiella pneumoniae ATCC 4352 & 4.3 & 22 & 49 & 99 \\
Staphylococcus aureus ATCC 6538 & 86 & 91 & 51 & 0 \\
\hline Escherichia coli & 13 & 35 & 0 & 37 \\
Enterococcus faecalis & 0 & 0 & 0 & 0 \\
\hline Staphylococcus saprophyticus & 0 & 0 & 0 & 0 \\
Pseudomonas aeruginosa & 0 & 0 & 21 \\
\hline
\end{tabular}

Table 2. Antimicrobial effect percentages of Nexus Energy Fiber ${ }^{\circledR}$ versus ordinary pure cotton as a function of time, in remote conditions.

\begin{tabular}{|c|c|c|c|c|}
\hline \multirow[t]{2}{*}{ Microorganisms } & \multicolumn{4}{|c|}{ Antimicrobial effect (\%) in function of time } \\
\hline & 0 hours & 6 hours & 24 hours & 48 hours \\
\hline Klebsiella pneumoniae ATCC 4352 & 0 & 0 & 0 & 100 \\
\hline Staphylococcus aureus ATCC 6538 & 0 & 0 & 24 & 100 \\
\hline Escherichia coli & 0 & 0 & 0 & 70 \\
\hline Pseudomonas aeruginosa & 0 & 0 & 23.73 & 75 \\
\hline
\end{tabular}

no effect on E. faecalis and S. saprophyticus; while it acts at a short contact time (6 hours) only against $S$. aureus. Remote conditions experiments showed an increase in the antimicrobial effect with the incubation time: compared to the direct contact cases, longer incubation times were however required before it became measurable. Particularly, 48 hours were needed to obtain a $100 \%$ effective antimicrobial response against $K$. pneumoniae and $E$. coli. For shorter contact times, no effect was observed. Lastly, NEF started showing a bactericidal activity contra $S$. aureus and $P$. aeruginosa after 24 hours only and it increased after 48 hours. Interestingly enough, no antimicrobial response was detected for the same incubation times in most of experiments performed in direct contact conditions. It should be noted that a significant drop in the antimicrobial effect occurs for the control ATCC strain $S$. aureus and the clinical $P$. aeruginosa isolate at a contact time of 24 hours. A bactericidal effect was instead observed for all strains in analysis after a 48-hour contact. It can therefore be concluded that even in remote conditions the NEF shows a bactericidal effect correlated to the contact time, with a relation of direct proportionality.

NEF shows a bactericidal effect on most of the tested microbial strains. Both the contact times and the distance play a significant role: in direct contact conditions, shorter contact times are required to measure antimicrobial effects, but a decrease in the response is observed at longer times for some strains ( $S$. aureus and E. coli). Instead, remote condition experiments show antimicrobial effects only at longer times but a direct proportionality with the contact time is always observed. These very preliminary results are encouraging: antibacterial properties of NEF deserve to be further tested.

\section{References}

1. ASTM. E-2180-07 (Reapproved 2012) Determining the activity of incorporated antimicrobial agents in polymeric or hydrophobic, Pesticides Antimicrobials, and Alternative Control Agents; Hazardous Substances and Oil Spill Response, Vol.11.08. ASTM Internat, West Conshohocken, PA, USA; 2012.

2. Irene $\mathrm{G}$, Georgios $\mathrm{P}$, Ioannis $\mathrm{C}$, et al. Copper-coated textiles: armor against MDR nosocomial pathogens. Diagn Microbiol Infect Dis 2016;85:2059.

3. Nexus Energy Fiber License, 2015.

4. Reichel M, Heisig P, Kampf G. Pitfalls in efficacy testing how important is the validation of neutralization of chlorhexidine digluconate? Ann ClinMicrobiol Antimicrob 2008;7:20. 